\title{
Impact of Fish Oil-Based Lipid Emulsion on Serum Triglyceride, Bilirubin, and Albumin Levels in Children With Parenteral Nutrition-Associated Liver Disease
}

\author{
SANG I. LEE, CLARISSA VALIM, PATRICK JOHNSTON, HAU D. LE, JONATHAN MEISEL, DANIELLE A. ARSENAULT, \\ KATHLEEN M. GURA, AND MARK PUDER
} Department of Surgery [S.I.L.], Harvard Medical School, Boston, Massachusetts 02115; Department of Surgery [C.V., P.J., H.D.L., J.M.,
D.A.A., K.M.G., M.P.], Children's Hospital Boston, Boston, Massachusetts 02115

\begin{abstract}
Parenteral nutrition is known to cause liver injury in babies. The aim of this study is to investigate the effects of different lipid emulsions on parenteral nutrition-associated cholestasis in infants. In addition, there may be a relationship between the lipid emulsion and triglyceride levels. Furthermore, triglyceride levels may correlate with direct bilirubin and albumin, as markers of liver impairment and nutritional status. Patients with parenteral nutritionassociated cholestasis who were treated with a fish oil-based lipid emulsion ( $n=18$ ) were prospectively followed for triglyceride, direct bilirubin, and albumin levels and compared with patients who were maintained on a soy-based lipid emulsion $(n=59)$. Triglyceride levels decreased in the fish oil cohort from a mean of $140 \mathrm{mg} / \mathrm{dL}$ at wk 0 to $40 \mathrm{mg} / \mathrm{dL}$ at wk 20 but remained unchanged at $\sim 140$ $\mathrm{mg} / \mathrm{dL}$ in the soybean cohort. Triglyceride levels of patients treated with fish oil declined over time, while those receiving soybean oil did not. Also, changes in triglyceride levels over time were directly correlated with direct bilirubin and inversely related to albumin levels. These findings may indicate an added benefit of reduced triglyceride levels for patients treated with fish oil and this effect coincides with markers for improved liver function and nutritional status. (Pediatr Res 66: 698-703, 2009)
\end{abstract}

$\mathrm{T}$ he ability to meet and sustain nutritional requirements is critically important in the pediatric population, more specifically neonates, where growth and normal development are essential components of life. Gastrointestinal impairment leading to malnutrition can result from anatomic constraints (e.g., short bowel syndrome) and/or functional malabsorption. Parenteral nutrition (PN) is the only tool available for the maintenance of patients unable to sustain nutritional needs enterally. Since its advent several decades ago, PN has been studied and modified to the point where now basic physiologic homeostasis is now attainable with $100 \%$ i.v. administration of carbohydrates, protein, and fat. However, with increased use, specifically in infants, pathologic consequences have also arisen. PN has long been known to cause devastating liver injury in babies, beginning with cholestasis and progressing to

Received April 10, 2009; accepted August 42009.

Correspondence: Sang Il Lee, M.D., Department of Surgery, Harvard Medical School, 11 Priscilla Road, No. 1, Boston, MA 02135; e-mail: slee10@bidmc.harvard.edu

Supported by NIH Grant DK069621-05; Office of Orphan Drug Products Development (FDA 1 R01 FD003460-01 and FDA 1 R01 FD003436-02); and the Children's Hospital Surgical Foundation. frank end-stage liver failure requiring liver transplantation or, in many cases, leading to death (1-4).

Direct bilirubin, as a surrogate for cholestasis, is a marker used to diagnose and monitor parenteral nutrition-associated liver disease in children. Cholestasis is a relative indicator of liver excretory function, reflected biochemically by plasma bilirubin, or more specifically direct bilirubin. A relative biochemical indicator of liver synthetic function is plasma albumin, which also serves as a marker for overall nutritional status and anabolic/catabolic balance. Together, direct bilirubin and albumin levels can be used to estimate degree of liver function and nutritional status.

PN itself is composed of carbohydrates, protein, and fat. The fat component is a vital source of noncarbohydrate calories and also prevents essential fatty acid deficiency. Standard i.v. lipid emulsions are soybean-based, which are mainly composed of linoleic acid (omega-6) with a small amount of alpha-linolenic acid (omega-3). There has been a recent surge in research interest regarding different types of fatty acids and implications on health care in general and liver disease specifically. Fish oil-based lipids, which contain mainly docosahexaenoic acid (omega-3) and eicosapentaenoic acid (omega-3) with small amounts of linoleic acid (omega-6) and arachadonic acid (omega-6), have been shown to be a more physiologically balanced fat source and to have anti-inflammatory potential. The benefits have been most definitively displayed in their cardioprotective effects $(5,6)$.

Our group has recently shown that substituting the conventional soybean-based emulsion with a fish oil-based emulsion may be effective in treating PN-associated cholestasis in infants $(7,8)$. We have also shown that fish oil-based lipid emulsion can be effective as a sole fat source in cases of hypertriglyceridemia or when standard soybean-based emulsions are contraindicated $(9,10)$. It should be noted that although both emulsions are primarily constituted of a single fatty acid type (omega-6 or omega-3), they are not a pure fatty acid subtype formulation and both possess some amount of the other (primarily omega-6 + some omega-3, primarily omega-3 + some omega-6).

Abbreviations: DB, direct bilirubin; IQR, interquartile range; PN, parenteral nutrition; TG, triglyceride 
In animal models, omega-3 fatty acid lipid emulsions prevented fatty liver disease, likely via improved triglyceride clearance coupled with anti-inflammatory properties (11). Triglycerides are made of a glycerol and three fatty acids and comprise almost all ingested fat. Triglyceride level is closely linked to that of cholesterol and has been indicated as a risk factor for atherosclerosis (12). Hypertriglyceridemia, or high plasma triglyceride levels, indicates a physiologic derangement in the balance of fat intake, storage, usage, and excretion, which itself is a marker of poor metabolic homeostasis (13).

The aim of this study is to investigate the effects of different lipid emulsions on parenteral nutrition-associated cholestasis in infants. On the basis of our previous work and clinical observations, we propose that there is a relationship between the source of lipid emulsion source and serum triglyceride levels. Furthermore, we hypothesize that triglyceride levels correlate with direct bilirubin and albumin, as general markers of liver function and nutritional status.

\section{MATERIALS AND METHODS}

Study cohort. From September 2004 to August 2006, 18 infants receiving PN with the conventional soybean-based lipid emulsion (Intralipid; Fresenius Kabi AG, Bad Homburg vdh, Germany) who developed cholestasis were treated with a fish oil-based lipid emulsion (Omegaven, Fresenius Kabi AG, Bad Homburg vdh, Germany) after informed consent was obtained. Treatment was authorized under a compassionate treatment protocol. After treatment, patients were prospectively followed. Eligibility criteria included direct bilirubin (DB) $>2 \mathrm{mg} / \mathrm{dL}$ and predicted duration of $\mathrm{PN}$ for $>30 \mathrm{~d}$ due to gastrointestinal disease. Children with other liver diseases, such as cystic fibrosis, inborn metabolic errors, and hepatitis $\mathrm{C}$, were excluded.

A contemporary historical cohort of 59 patients who developed cholestasis while receiving conventional soybean emulsion with PN between February 2000 and January 2006 at Children's Hospital Boston was identified for comparison. These patients were selected after reviewing the records of 2125 patients who received PN during this period, according to pharmacy billing records. Eligibility criteria included diagnosis of gastrointestinal disease, age $<2 \mathrm{y}$, cholestasis secondary to PN (two consecutive DB $>2 \mathrm{mg} / \mathrm{dL}$ ), and predicted duration of $\mathrm{PN}>30 \mathrm{~d}$ at the time that cholestasis started. Children with cholestasis that was not exclusively due to $\mathrm{PN}$-associated liver damage and children whose medical records at Children's Hospital Boston provided insufficient data for analysis were excluded (2066 patients).

Study treatment. In the fish oil cohort, the soybean oil-based lipid emulsion was discontinued and treatment with fish oil-based lipid emulsion was started. For the first $2 \mathrm{~d}$ of treatment, patients received fish oil emulsions at 0.5 $\mathrm{g} \cdot \mathrm{kg}^{-1} \cdot \mathrm{d}^{-1}$ and were progressed to the maintenance dosage of 1 $\mathrm{g} \cdot \mathrm{kg}^{-1} \cdot \mathrm{d}^{-1}$ over for $12 \mathrm{~h}$. Dosing was based on previous usage of fish oil emulsion as monotherapy. The comparison group received a soybean-based lipid emulsion with doses ranging from 1 to $4 \mathrm{~g} \cdot \mathrm{kg}^{-1} \cdot \mathrm{d}^{-1}$ over for $24 \mathrm{~h}$.

Follow-up. Clinical and laboratory values were recorded prospectively in the fish oil cohort and retrospectively in the soybean cohort. For the fish oil cohort, when patients had more than one weekly data point, the weekly mean was estimated. For the soybean cohort, when patients had more than one weekly data point, the dates with the most data points were preferentially used. Baseline was defined as the date that administration of Omegaven began (or the previous week) for the fish oil cohort and the date of the second consecutive weekly DB $>2 \mathrm{mg} / \mathrm{dL}$ (or the previous week) for the soybean cohort. In both the fish oil and the soybean cohorts, patients were followed for a maximum time of $19 \mathrm{wk}$. After this time, only two patients, potentially different from the majority of patients, remained in the fish oil cohort. However, conclusions were comparable when analyzing the entire available follow-up period of these patients who remained in the cohort after wk 19 (data not shown).

Statistics. Statistical significance of baseline differences between the two groups was assessed using Wilcoxon tests, when reporting medians, and $\chi^{2}$ tests, when reporting proportions (or Fisher's exact tests, when warranted). All three laboratory tests of interest [triglyceride (TG), DB, albumin] were logarithmically transformed (natural logarithm) to assure validity of statistical assumptions (symmetry and linear functional association between tests and weeks time in regression analysis). DB of zero was assumed to be 0.1 . All the three tests were imputed if two consecutive values were missing by linearly interpolating the two closest values. A total of $768 \mathrm{DB}$ values were recorded between wk 0 and 19: 222 in the prospectively studied fish oil cohort and 546 in the retrospectively studied conventional lipid cohort. Chances of missing DB in both cohorts were small and comparable: $6 \%$ in the fish oil cohort $(14 /[222+14])$ and $9 \%$ in the conventional lipid cohort $(55 /[55+546])$. We imputed 14 missing records in the fish oil cohort (100\% of the 14 missing) and 55 in the conventional lipid cohort (100\% of the 55 missing). A total of 586 TG values were recorded between wk 0 and 19: 209 in the fish oil cohort and 377 in the conventional lipid cohort. Chances of missing TG in the fish oil cohort $(11 \%, 27 /[209+27])$ were smaller than in the conventional lipid cohort $(37 \%$, 224/[377 + 224]). We imputed 19 missing records in the fish oil cohort (70\% of the 27 missing) and 82 in the conventional lipid cohort (37\% of the 224 missing). Albumin was recorded only in the fish oil cohort: 21 observations with missing levels and 215 with recorded albumin levels (9\% chance of missing albumin). We imputed a total of 15 albumin values (71\% of the 21 missing). Because chances of missing TG levels were lower in the fish oil cohort than in the conventional lipid cohort, we performed a sensitivity analysis of missingness of TG by comparing analyses without imputation, with local imputation of two consecutive values (via interpolation), and with imputation of all values (via interpolation). Conclusions were comparable in all cases; thus, we present results of analyses based on linear interpolation of two consecutive values.

Changes in TG levels in the fish oil and conventional lipid cohorts were described via the weekly geometric mean across all subjects. The geometric mean corresponds to the exponential of the mean of the natural logarithm of a variable. Comparisons of the mean trajectories over time between the two study cohorts were performed using mixed-effect models including a random intercept and slope for week. In this analysis, the covariance of coefficients was assumed to be unstructured and the variance of the residuals was based on the power (to week) function. The choice of the functional form between week and mean TG was based on a $p<0.05$ of the likelihood ratio test (LRT) of second or higher order polynomial coefficients for week and on Akaike's Information Criterion (AIC). Decisions about choices of random effect and other terms of the covariance matrix were based on restricted maximum likelihood (REML) and AIC. The adjusted effect of the fat emulsion was also estimated in models including treatment effect and the following potential confounders: gestational age, birth weight, diagnosis of necrotizing enterocolitis, number of diagnoses (1 versus 2 or more), age at baseline, and duration of PN before baseline. In adjusted models, we forced in age at baseline and included any additional covariates that appreciably affected the coefficient of treatment. Age at baseline is a surrogate for several risk factors of liver disease in patients receiving PN and was statistically significantly different across the two cohorts at baseline.

To study the association of the trajectory of TG, DB, and albumin over time, weekly geometric means were inspected and individual concurrent correlation coefficients were estimated separately in the fish oil and conventional lipid cohorts except for albumin, which was available only in the fish oil cohort. Pearson pairwise correlation coefficients between each two tests were estimated for each patient across weeks. The distribution of the individual correlation coefficients was graphically examined and a weighted mean across patients was estimated with weights based on the number of weeks each patient contributed for the correlation. Confidence intervals for correlation coefficients were estimated using weighted least squares of the FisherZ-transformed individual coefficients. When estimating correlation coefficient, patients who only had one or two pairs of measurement (correlation of + or -1$)$ were excluded. It is important to note that correlation coefficients assuming independence of the observations across time were approximately equal to the weighted mean of individual correlation coefficients. In addition, besides concurrent correlations, we estimated correlation coefficients for lagged $(1,2$, and $3 \mathrm{wk})$ tests and obtained results comparable with the concurrent analysis.

Throughout the results, we will refer to the geometric mean of TG, DB, and albumin simply as means. All $p$ values were two-sided. Analyses were performed in SAS 9.1 (SAS Institute Inc, Cary, NC).

United States Food and Drug Administration approval was obtained before administration of Omegaven. The Children's Hospital Boston Institutional Review Board also approved this study and informed consent was obtained.

\section{RESULTS}

Demographics. Children in both the groups were similar in demographic and clinical characteristics at baseline, except for age (Table 1). Median age was 13 and $7 \mathrm{wk}$ in the fish oil and 
Table 1. Baseline characteristics*

\begin{tabular}{|c|c|c|c|}
\hline Variables & Fish oil $(n=18)$ & Soybean oil $(n=59)$ & $p$ Value $\dagger$ \\
\hline \multicolumn{4}{|l|}{ Demographic } \\
\hline Gender (male), $n(\%)$ & $12(67 \%)$ & $35(59 \%)$ & 0.58 \\
\hline Age (wk), median (IQR) & $13(8,18)$ & $7(5,12)$ & 0.003 \\
\hline Birth weight (kg), median (IQR) & $1.30(1.10,2.26)$ & $1.57(0.97,2.30)$ & 0.95 \\
\hline Gestational age (wk), mean $\pm \mathrm{SD}$ & $31(27,34)$ & $32(28,36)$ & 0.14 \\
\hline \multicolumn{4}{|l|}{ Ethnicity, $n(\%) \ddagger$} \\
\hline African-American & $5(29 \%)$ & $10(24 \%)$ & $0.69 \S$ \\
\hline Caucasian or Asian & $10(59 \%)$ & $26(62 \%)$ & \\
\hline Latino & $2(12 \%)$ & $3(7 \%)$ & \\
\hline \multicolumn{4}{|l|}{ Clinical } \\
\hline Duration of PN (wk), median (IQR) & $8(5,18)$ & $7(5,19)$ & 0.74 \\
\hline Diagnosis of gastroschisis, $n(\%)$ & $4(22 \%)$ & $11(19 \%)$ & 0.74 \\
\hline Diagnosis of intestinal atresia, $n(\%)$ & $6(33 \%)$ & $11(19 \%)$ & 0.19 \\
\hline Diagnosis of malrotation/midgut volvulus, $n(\%)$ & $3(17 \%)$ & $6(10 \%)$ & $0.43 \S$ \\
\hline Diagnosis of necrotizing enterocolitis, $n(\%)$ & $10(56 \%)$ & $26(44 \%)$ & 0.39 \\
\hline Other diagnosis, $n(\%) \|$ & $3(17 \%)$ & $35(59 \%)$ & $0.002 \S$ \\
\hline \multicolumn{4}{|l|}{ Number of diagnoses, $n(\%)$} \\
\hline 1 & $11(61 \%)$ & $27(47 \%)$ & $0.38 \S$ \\
\hline 2 & $6(33 \%)$ & $28(49 \%)$ & \\
\hline 3 & $1(6 \%)$ & $2(4 \%)$ & \\
\hline \multicolumn{4}{|l|}{ Laboratory } \\
\hline Direct bilirubin (mg/dL), median (IQR) & $5.4(3.4-6.9)$ & $4.3(3.3-6.6)$ & 0.33 \\
\hline Triglycerides (mg/dL), median (IQR) & $153.4(99,183)$ & $130(92,176)$ & 0.39 \\
\hline ALT (IU/dL), median (IQR) & $96(69,168)$ & $48(26,120)$ & 0.18 \\
\hline Platelets, median (IQR) & $208(104,252)$ & $261(105-378)$ & 0.14 \\
\hline INR, median (IQR)凹 & $1.1(1.0,1.2)$ & $1.3(1.0,1.7)$ & 0.13 \\
\hline PT (s), median (IQR)I & $12(11,12)$ & $14(11,16)$ & 0.12 \\
\hline PTT (s), median (IQR)II & $35(31,18)$ & $31(23,34)$ & 0.77 \\
\hline
\end{tabular}

ALT, alanine aminotransferase; INR, international normalized ratio; PT, prothrombin time; PTT, partial thromboplastin time.

$*$ Baseline is defined as the date that treatment started for Omegaven patients and the date that the second of two consecutive direct bilirubins were $>2$ mg/dL for Intralipid patients.

$\dagger p$ values for continuous variables were obtained via Wilcoxon test and $p$ values for differences of proportions were obtained via Pearson's chi-square test, except when marked with $\S$, where Fisher's exact test was used.

$\ddagger$ There were three subjects of other ethnicity in the soybean oil cohort.

\| Other diagnoses included bowel obstruction, Christmas tree intestinal atresia, omphalocele, and Hirschsprung's disease.

II Few subjects had information about INR, PT, and PTT at baseline. INR was recorded for 10 fish oil and 11 soybean oil patients and PT/PTT were recorded for 10/11 fish oil and 12/12 soybean oil patients, respectively.

conventional lipid cohorts, respectively, $(\mathrm{P}-\mathrm{Wilcoxon}=$ 0.003). From baseline until wk 19, the fish oil cohort was followed for a median of $14 \mathrm{wk}$ (interquartile range [IQR] = $11,18 \mathrm{wk})$ and the conventional emulsion cohort was followed for $15 \mathrm{wk}$ (IQR = 7, 19 wk). By wk 19, 11 patients $(61 \%)$ in the fish oil cohort had completed follow-up: eight had been weaned off PN, two had expired, and 16 had reached $\mathrm{DB}<2 \mathrm{mg} / \mathrm{dL}$. In the conventional lipid cohort, 24 patients (41\%) had completed follow-up: 40 had been weaned off PN, 12 had expired, six had undergone transplantation, and 28 had reached $\mathrm{DB}<2 \mathrm{mg} / \mathrm{dL}$.

TG levels in the fish oil versus soybean oil cohorts. TG levels decreased in the fish oil cohort from a mean of 140 $\mathrm{mg} / \mathrm{dL}$ at wk 0 (or baseline) to $40 \mathrm{mg} / \mathrm{dL}$ at wk 20 but remained at $\sim 140 \mathrm{mg} / \mathrm{dL}$ throughout the study period in the conventional soybean oil cohort (Fig. 1). Differences in the trajectories of mean weekly TG levels between fish oil and soybean oil cohorts were statistically significant $(p<0.001$; Fig. 1). The observed weekly mean TG levels of the two cohorts suggested that $8 \mathrm{wk}$ passed before TG levels started decreasing (Fig. 2). Conclusions about differences in TG trajectories between the two cohorts were comparable in unadjusted and adjusted analysis (Table 2). In unadjusted

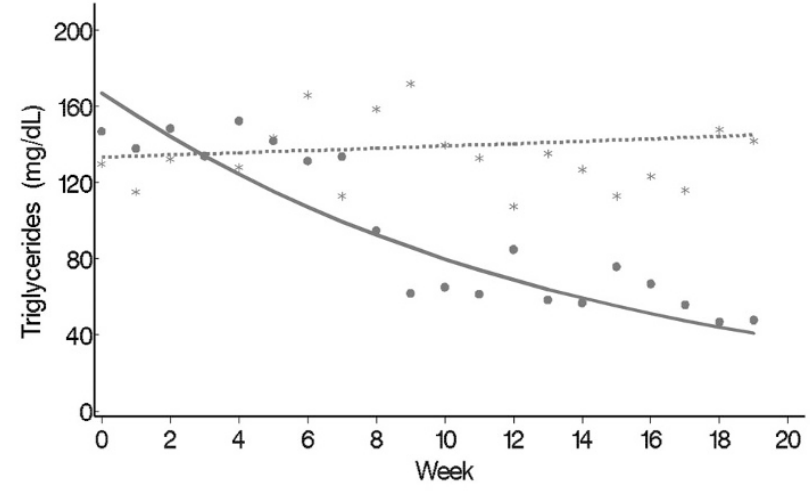

Figure 1. Observed weekly mean (fish oil $=$ dots, soybean oil $=$ stars) and mean trajectory over weeks, estimated in a random effects model (fish oil = solid line, soybean oil $=$ dashed line) by the type of IV fat emulsion. Means represent geometric means or exponential $\{$ mean[ln (test)]\}. $\mathrm{P}$ represents $p$ value for testing difference in mean trajectory of triglyceride (TG) over weeks between fish oil and soybean oil in the following random effects model: 4.89 +0.23 fish oil $(v s$ soybean oil $)+0.004$ wk -0.08 fish oil $\times$ week.

analysis, the weekly decline in the mean logarithm of TG levels in the fish oil cohort was $-0.07(95 \% \mathrm{CI}=-0.10$, $-0.05)$, and the mean increase in the soybean oil cohort was 


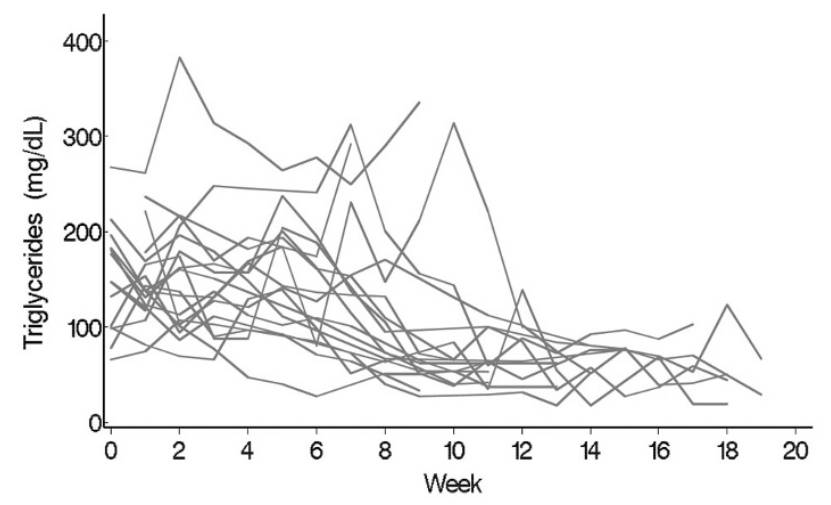

Figure 2. Individual trajectory of triglyceride (TG) after beginning fish oil until wk 19 .

Table 2. Unadjusted and adjusted estimates of the difference between TG decline over time in fish oil and soybean oil cohorts, obtained in random effect models*

\begin{tabular}{lcl}
\hline \multicolumn{1}{c}{ Predictor } & $\begin{array}{c}\text { Change in logarithm } \\
\text { of TG }(\mathrm{mg} / \mathrm{dL})\end{array}$ & \multicolumn{1}{c}{$95 \% \mathrm{CI}$} \\
\hline Unadjusted model $\dagger$ & & \\
Time in the fish oil cohort & -0.07 & $(-0.10,-0.05)$ \\
Time in the soybean oil cohort & 0.004 & $(-0.01,0.02)$ \\
Adjusted model $\neq$ & & \\
Time in the fish oil cohort & -0.07 & $(-0.10,-0.05)$ \\
Time in the soybean oil cohort & 0.005 & $(-0.01,0.02)$ \\
Age & 0.02 & $(0.01,0.02)$ \\
\hline
\end{tabular}

* Random effect models included a random intercept and a random slope for the effect of time. Also, covariance of coefficients was assumed to be unstructured and residual variance was assumed to follow a power of week function. In both unadjusted and adjusted models, the $p$ value for the interaction between type of IV fat and week was $<0.001$.

$\dagger$ Crude model: $4.89+0.23$ fish oil $(v s$ soybean oil $)+0.004$ week -0.08 fish oil $x$ week.

† Adjusted model: $4.68+0.18$ fish oil $(v s$ soybean oil) +0.005 week 0.08 fish oil $\times$ week +0.02 age at baseline.

$0.005(95 \% \mathrm{CI}=-0.009,0.018)$. In other words, the percent change in TG levels from wk 0 to wk 10 in the fish oil cohort was $-50 \%(95 \% \mathrm{CI}=-63 \%,-39 \%)$ and in the soybean oil cohort was $4.1 \%(95 \% \mathrm{CI}=-9.5 \%, 22 \%)$. No covariate appreciably affected the effect of type of fat emulsion on TG trajectories over time. The association between age and TG trajectories over time suggested that TG levels were higher at wk 0 in older children.

Correlation of TG, DB, and albumin. Declines in TG and DB levels over time were positively associated in the fish oil cohort (Fig. 3). In the soybean oil cohort, while mean DB levels declined, TG levels remained unchanged (Fig. 4). Mean curves of TG and albumin levels in the fish oil cohort were inversely associated; when TG levels started decreasing, albumin started increasing. The association between DB and albumin was also inversely associated and when DB decreased to levels $\leq 2 \mathrm{mg} / \mathrm{dL}$, albumin started increasing in the fish oil cohort. The weighted mean of the individual correlation coefficients of TG with DB also suggested a strong positive correlation between the two markers in the fish oil cohort $(0.70 ; 95 \% \mathrm{CI}=0.63,0.86)$. In the soybean oil cohort, the correlation was low to moderate $(0.31 ; 95 \% \mathrm{CI}=0.18$,

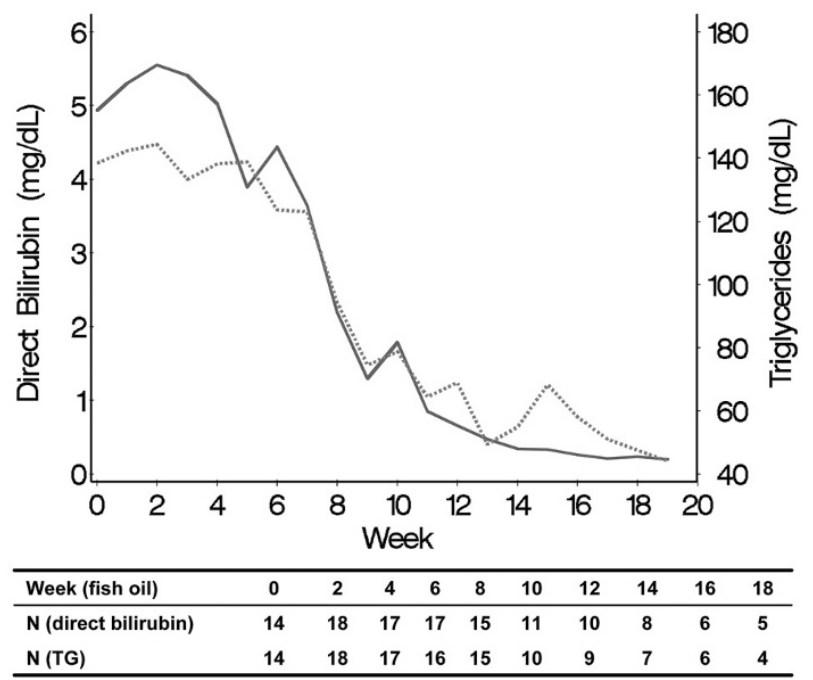

Figure 3. Pairwise associations of observed weekly mean trajectories of direct bilirubin (solid line) and triglyceride (TG) (line with points), representing the association between direct bilirubin and TG in the fish oil cohort. Numbers included in the weekly mean estimation for each laboratory test are shown.

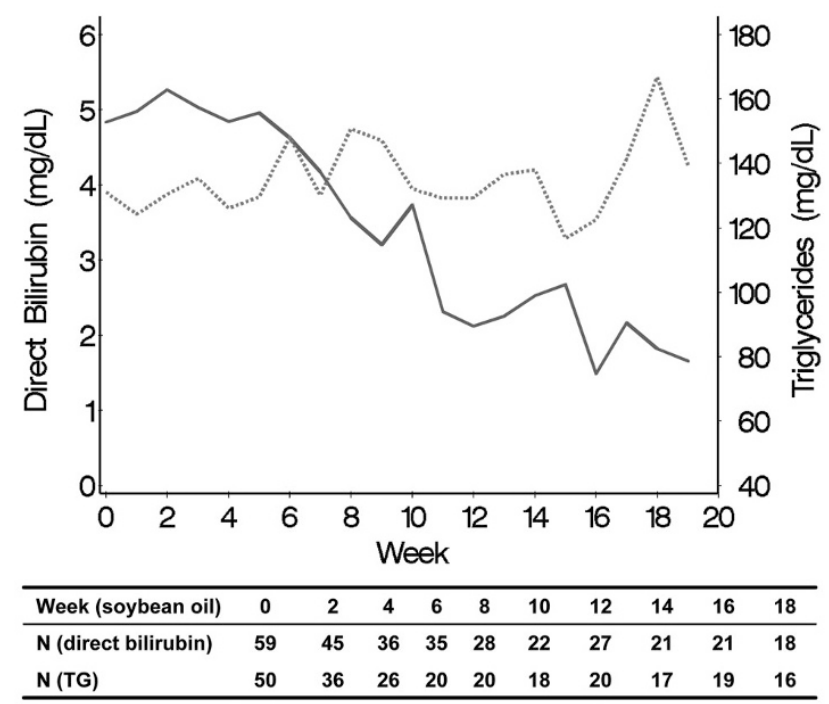

Figure 4. Pairwise associations of observed weekly mean trajectories of direct bilirubin (solid line) and triglyceride (TG) (line with points), representing the association between direct bilirubin and TG in the soybean oil cohort. Numbers included in the weekly mean estimation for each laboratory test are shown.

0.54) but also positive, contrary to previously stated data (Fig. 4). It is possible that the correlation coefficients are overestimating the true correlation; the correlation coefficient may be over-representing the trajectory before wk 12 , since it is based on the weeks in which both DB and TG are measured. In the retrospective soybean oil cohort, after DB reached levels $>2$ $\mathrm{mg} / \mathrm{dL}$, TG levels were measured less often $(21 \% \mathrm{TG}$ were missing among 515 wk with $\mathrm{DB}>2$ ) compared with weeks in which DB $\leq 2$ ( $40 \%$ TG were missing among 86 wk with DB $>2$ ). Overall, individual correlations between TG and DB in the fish oil cohort were strong, although one subject had an inverse (negative) correlation (Fig. 7). Individual's correla- 
tions in the fish oil cohort between TG and albumin and between DB and albumin were low to moderate and inverse, respectively. Among three subjects whose correlation was opposite to the correlation in the rest of the fish oil cohort, none were weaned off PN and 33\% (1) died, as opposed to all other 15 subjects in which 53\% were weaned off PN and $13 \%$ died, suggesting that patients with worse liver disease may exhibit poorer correlations.

\section{DISCUSSION}

Epidemiologic studies have shown that before the age of food industrialization, humans subsisted on an omega- 6 to omega-3 fatty acid ratio of $\sim 1: 1$. Currently, that ratio stands at 16:1 in modern Western societies $(14,15)$. Over large periods of time, it is logical to believe that this would have long-lasting effects on disease prevalence and indeed, diseases such as atherosclerosis, heart disease, obesity, and diabetes have sharply increased during the past century. Admittedly, dietary factors and their impact on all-encompassing disease prevalence and severity are unknown, but it would be inappropriate to discount their influence. On the basis of this overarching theory and basic science data that has repeatedly demonstrated the benefits of omega- 3 fatty acids in a number of disease processes $(5,16)$, we believe that substituting fish oil-based lipid emulsions for the standard soybean-based emulsion, which has been used for the majority of the existence of PN, will result in normalizing the severely deranged physiologic balance that exists in patients dependent on PN to some extent. These derangements manifest as poor glycemic control, cholestasis, possibly a low-level ubiquitous inflammatory state, and hypertriglyceridemia $(5,6)$. We have previously shown that a fish oil-based lipid emulsion can be used safely and effectively in $\mathrm{PN}$-associated cholestasis in children $(7,8)$, but we believe that the benefits go beyond this. This study is an attempt to display another benefit of this treatment modality in decreasing TG levels and correlating these findings with other parameters to show a more global beneficial effect.

TG levels in patients on i.v. fish oil decline, or decline more rapidly, over time, whereas TG levels in patients on i.v. soybean oil remain the same, or decline more slowly (Fig. 1). Supplementation with fish oil, as opposed to soybean oil, presents a more physiologically sound source of fat in that it provides a less inflammatory substrate for the inflammatory cascade and it promotes fat secretion and processing (17-19). This results in a more efficient metabolism, possibly reflected by a decreased number of circulating TG.

Furthermore, these changes correlate directly with DB levels (Figs. 3, 4, and 7). Omega-3 fatty acid-mediated decrease in cholestasis, specifically improved bile excretion, results in improved lipid metabolism and hence, decreased TG levels $(11,20,21)$. The direct relationship observed between DB and TG levels is likely more a reflection of improved liver function leading to better lipid metabolism than it is a reflection of a bile-mediated mechanism, seeing how most of these patients are taking minimal enteral nutrition. Bile assists in the enteral absorption of fat, which is bypassed by i.v. administration.

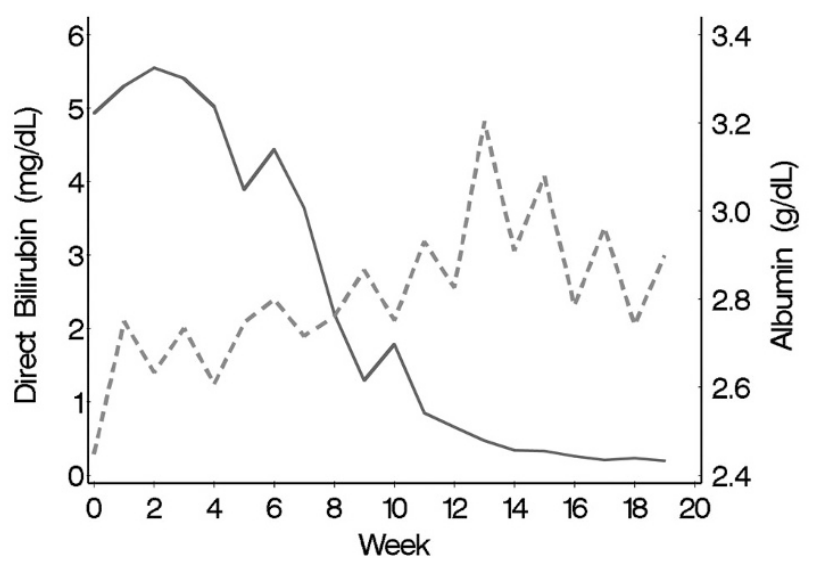

Figure 5. Pairwise associations of observed weekly mean trajectories of direct bilirubin (solid line) and albumin (dashed line), representing the association between direct bilirubin and albumin in the fish oil cohort.

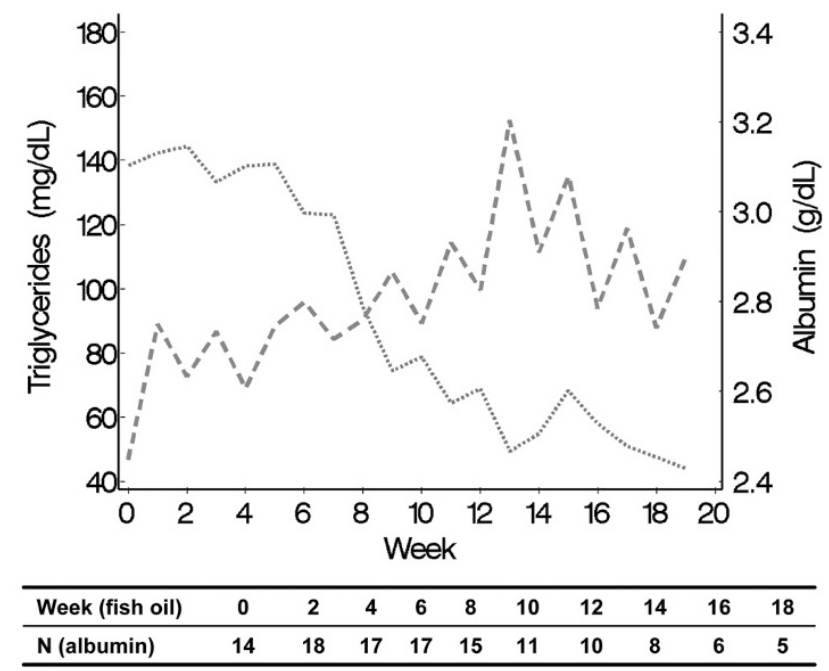

Figure 6. Pairwise associations of observed weekly mean trajectories of triglyceride (TG) (line with points) and albumin (dashed line), representing the association between TG and albumin in the fish oil cohort. Numbers included in the weekly mean estimation for each laboratory test are shown.

Albumin was shown to be inversely related to TG levels in the fish oil cohort (Figs. 5-7). Increased levels of albumin generally indicate adequate nutrition via protein turnover shifted toward an anabolic state. In addition, it can also reflect the synthetic function of the liver. Concurrent increase of albumin with decrease in TG and DB levels lends further evidence to the overall beneficial effects that fish oil supplementation has on liver processes and nutrition.

Parenteral omega- 6 fatty acids, in the form of soybean oil, may be more toxic than once thought, perhaps contributing to $\mathrm{PN}$-associated liver disease, hypertriglyceridemia, and an overall inflammatory state in infants that predisposes to further physiologic insults $(5,17)$. We propose that substituting the soybean-based lipid emulsion with a fish oil-based lipid emulsion can provide the same benefits, namely high-density calories and prevention of essential fatty acid deficiency, without the potential drawbacks. The data presented suggests that fish oil supplementation in PN-dependent infants results 


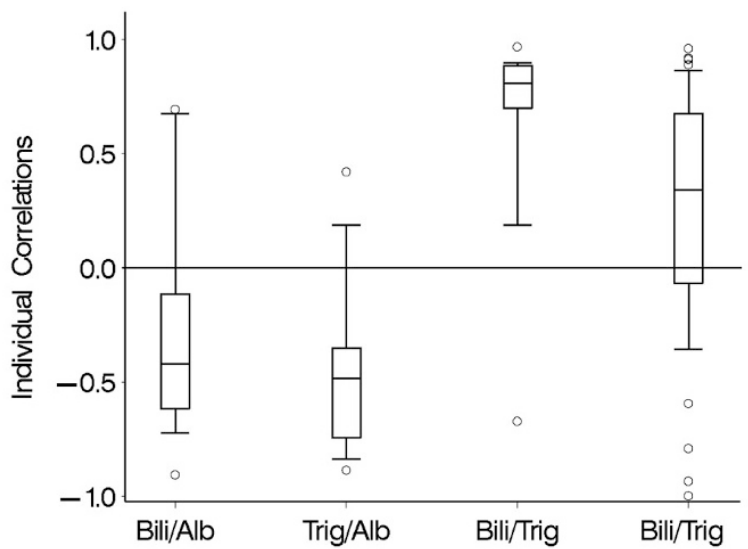

Figure 7. Distribution of individual Pearson correlation coefficients for pairwise association of triglyceride (TG), direct bilirubin, and albumin in the fish oil cohort and TG and albumin in the soybean oil cohort. The solid bar within the box represents the median value; upper boundary, 75th percentile; lower boundary, 25th percentile; whiskers extend from the 10th to 90th percentiles. Correlation of TG with direct bilirubin in the soybean oil cohort excluded six subjects who had one or less pair of observations and 12 subjects who had two pairs of observations (nine with correlation +1 and three with correlation -1).

in improved lipid metabolism, which correlates with improved liver function and nutrition.

Observed clinical effects may be more attributable to the fish oil-based lipid emulsion rather than omega- 3 fatty acids themselves. The fact that the fish oil-based lipid emulsions possess a certain amount of omega- 6 fatty acids makes the latter conclusion tenuous. In addition, the soybean-based lipid emulsion does possess a certain amount of downstream omega-3 fatty acids. Although strongly suggested given the proportion of the fatty acid subtypes, definitive studies examining pure lipid emulsions may be necessary to achieve a direct clinical correlation. The potential mechanism of these results may be that the positive fish oil-derived effects require a threshold concentration that is not achieved by the trace amounts of omega-3 fatty acids seen in the soybean-based lipid emulsion.

Prospective randomized data are currently being collected, which will further elucidate the relationship between fish oil-based lipid emulsions and cholestasis and TG levels in PN-dependent infants.

\section{REFERENCES}

1. Buchman A 2002 Total parenteral nutrition-associated liver disease. JPEN J Parenter Enteral Nutr 26:S43-S48

2. Freund HR 1991 Abnormalities of liver function and hepatic damage associated with total parenteral nutrition. Nutrition 7:1-5; discussion 5-6

3. Kubota A, Yonekura T, Hoki M, Oyanagi H, Kawahara H, Yagi M, Imura K, Iiboshi Y, Wasa K, Kamata S, Okada A 2000 Total parenteral nutrition-associated intrahepatic cholestasis in infants: 25 years' experience. J Pediatr Surg 35:1049-1051

4. Mullick FG, Moran CA, Ishak KG 1994 Total parenteral nutrition: a histopathologic analysis of the liver changes in 20 children. Mod Pathol 7:190-194

5. Lee S, Gura KM, Kim S, Arsenault DA, Bistrian BR, Puder M 2006 Current clinica applications of omega-6 and omega-3 fatty acids. Nutr Clin Pract 21:323-341

6. Lee S, Gura KM, Puder M 2007 Omega-3 fatty acids and liver disease. Hepatology 45:841-845

7. Gura KM, Lee S, Valim C, Zhou J, Kim S, Modi BP, Arsenault DA, Strijbosch RA, Lopes S, Duggan C, Puder M 2008 Safety and efficacy of a fish-oil-based fat emulsion in the treatment of parenteral nutrition-associated liver disease. Pediatrics 121:e678-e686

8. Gura KM, Duggan CP, Collier SB, Jennings RW, Folkman J, Bistrian BR, Puder M 2006 Reversal of parenteral nutrition-associated liver disease in two infants with short bowel syndrome using parenteral fish oil: implications for future management. Pediatrics 118:e197-e201

9. Gura K, Strijbosch R, Arnold S, McPherson C, Puder M 2007 The role of an intravenous fat emulsion composed of fish oil in a parenteral nutrition-dependent patient with hypertriglyceridemia. Nutr Clin Pract 22:664-672

10. Gura KM, Parsons SK, Bechard LJ, Henderson T, Dorsey M, Phipatanakul W, Duggan C, Puder M, Lenders C 2005 Use of a fish oil-based lipid emulsion to treat essential fatty acid deficiency in a soy allergic patient receiving parenteral nutrition. Clin Nutr 24:839-847

11. Alwayn IP, Gura K, Nose V, Zausche B, Javid P, Garza J, Verbesey J, Voss S, Ollero M, Andersson C, Bistrian B, Folkman J, Puder M 2005 Omega-3 fatty acid supplementation prevents hepatic steatosis in a murine model of nonalcoholic fatty liver disease. Pediatr Res 57:445-452

12. Cullen $P 2000$ Evidence that triglycerides are an independent coronary heart disease risk factor. Am J Cardiol 86:943-949

13. Yuan G, Al-Shali KZ, Hegele RA 2007 Hypertriglyceridemia: its etiology, effects and treatment. CMAJ 176:1113-1120

14. Simopoulos AP 1993 n-3 fatty acid-enriched eggs, lipids, and Western diet: time for change. Nutrition 9:561-562

15. Simopoulos AP 1999 Evolutionary aspects of omega-3 fatty acids in the food supply. Prostaglandins Leukot Essent Fatty Acids 60:421-429

16. Kris-Etherton PM, Harris WS, Appel LJ; AHA Nutrition Committee. American Heart Association 2003 Omega-3 fatty acids and cardiovascular disease: new recommendations from the American Heart Association. Arterioscler Thromb Vasc Biol 23:151-152

17. Grimminger F, Wahn H, Mayer K, Kiss L, Walmrath D, Seeger W 1997 Impact of arachidonic versus eicosapentaenoic acid on exotonin-induced lung vascular leak age: relation to 4-series versus 5-series leukotriene generation. Am J Respir Crit Care Med 155:513-519

18. Leaf A, Weber PC 1988 Cardiovascular effects of n-3 fatty acids. N Engl J Med 318:549-557

19. Schmidt EB, Dyerberg J 1994 Omega-3 fatty acids. Current status in cardiovascular medicine. Drugs 47:405-424

20. Araya J, Rodrigo R, Videla LA, Thielemann L, Orellana M, Pettinelli P, Poniachik J 2004 Increase in long-chain polyunsaturated fatty acid $n-6 / n-3$ ratio in relation to hepatic steatosis in patients with non-alcoholic fatty liver disease. Clin Sci (Lond) 106:635-643

21. Van Aerde JE, Duerksen DR, Gramlich L, Meddings JB, Chan G, Thomson AB, Clandinin MT 1999 Intravenous fish oil emulsion attenuates total parenteral nutrition-induced cholestasis in newborn piglets. Pediatr Res 45:202-208 